\title{
Wireless Technologies in Factory Automation
}

\author{
Aurel Buda, Volker Schuermann and Joerg F. Wollert \\ University of Applied Sciences Bochum
}

Germany

\section{Introduction}

Wireless technologies are utilised in consumer applications for several years, now. However, the operation of radio based communication systems in automation applications was considered doubtful for a long time. Primarily, the highly fluctuating quality of wireless transmission channels, compared to wired ones, was responsible for this fact. Transmitted electromagnetic waves experience reflexion, scattering, and diffraction, which may cause constructive or destructive interferences of the different signal copies arriving at the receiver. The direct consequences are packet errors and losses, resulting in higher transmission delays. Especially industrial propagation environments, with a lot of metallic surfaces and moving objects, are classified as demanding for wireless transmission. While fluctuations in latency and short losses of connections may be tolerated in consumer applications, exceeding given timelines in automation applications implies intolerable errors. The results are low plant availabilities and decreasing productivity.

By means of the development of diverse wireless standards and the adaption of well-suited protocols for industrial applications, the end-users doubts could be reduced dramatically over the last decay. The advantages of wireless solutions are obvious. In harsh environments, mobile and rotating scenarios, or at positions, difficult to access, cable connections and sliding contacts represent a main source of error. In this context the error probability can be decreased and the maintenance intervals increased by the utilisation of wireless technologies. In addition to that, there is a great potential on saving time and money during planning, installation, and commissioning of plant sections. Many domains of the industrial automation already profit from the deployment of wireless solutions. With KNX RF (Konnex, 2006) and ZigBee (ZigBee Standards Organization, 2007) the first standards for building automation have been introduced. Within the scope of the HART 7 specifications (HART Communication Foundation, 2008) the first standard, WirelessHART, for the process automation was released in late 2007. Further standards, especially for the domain of factory automation, are expected to get published in 2010. In order to reduce costs and time during the development of wireless solutions, unlicensed frequency bands are typically used for operation. Moreover an almost worldwide harmonised operation is guaranteed. This tendency is very pronounced for the $2.45 \mathrm{GHz}$ ISM (Industrial, Scientific, and Medical) frequency band. Because of the high availability, transceiver chips of commercial standards are 
often applied on the physical layer. Good examples are the technologies of IEEE 802.11 (LAN/MAN Standards Committee of the IEEE Computer Society, 2007a), IEEE 802.15.1 (LAN/MAN Standards Committee of the IEEE Computer Society, 2005)/Bluetooth or IEEE 802.15.4 (LAN/MAN Standards Committee of the IEEE Computer Society, 2006). Previous analysis (Willig et al., 2005; Vedral et al., 2006; Vedral \& Wollert, 2006) attested the capabilities of these technologies for industrial applications. In order to comply with the requirements in automation with respect to determinism, reliability, and availability, above the physical layer adapted protocols are implemented. With latency requirements of $100 \mathrm{~ms}$... $<1 \mathrm{~ms}$, the domain of factory automation is one of the most demanding. With state of the art technologies, wireless solutions may serve applications with update times of $10 \mathrm{~ms} . .20 \mathrm{~ms}$. Below these timelines the reserve for packet retransmissions and error corrections of the current technologies is insufficient to guarantee a reliable communication. The upcoming ultra wideband (UWB) technologies are considered potential candidates for faster wireless solutions of the next generation. Within the scope of the chapter "Wireless Technologies for Factory Automation" the current state of the art and research of wireless technologies in factory automation gets introduced.

The rest of the chapter is organised as follows. Section 2 gives a short overview of the requirements of wireless technologies in factory automation and its possible applications. In Section 3 the properties of wireless transmission channels with respect to industrial environments are depicted. In addition to that, performance increasing techniques are stated, as well. Section 4 describes the state of the art of wireless base technologies in fabric automation, their utilisation and the regulation for the $2.45 \mathrm{GHz}$ ISM frequency band. Furthermore the impact of coexisting wireless technologies and the necessity for coexistence management are discussed. Section 5 gives an overview of upcoming wireless technologies and the frequency regulation for UWB devices. Section 6 gives a conclusion of the most important information.

Due to the large span of the topic, this chapter can only give an overview of the recent properties and technologies. For further insights the most important references of each subject are listed.

\section{Performance Requirements of Communication Systems in Factory Automation}

Figure 1 shows the hierarchical model of automation. With respect to the current state of the art, wireless technologies may be applied, as a supplement to existing wired communication networks, across all levels of the model. The major part of automation devices is equipped on the control and sensor/actuator level.

Basically, the control level consists of programmable logic controllers (PLCs) and (intelligent) decentralised I/O-Terminals, which are interconnected by one or more field buses (IEC, 2007a). Depending on the plant, the number of devices varies and their distances may range from a few to some hundreds of meters. Classical field buses for example are Profibus or Interbus (IEC, 2007b). Current field buses aim at convergence to the well established Ethernet IT-networks with data rates of up to $100 \mathrm{Mbps}$. Popular examples are Profinet, EtherCat, Ethernet-Powerlink, Sercos III and Ethernet/IP (IEC, 2007c). Depending on the area of application typical cycle times of $10 \mathrm{~ms} . .100 \mathrm{~ms}$ (PLC to PLC, Visualization), 
$1 \mathrm{~ms} . .10 \mathrm{~ms}$ (Communication to decentralized peripherals), and $<1 \mathrm{~ms}$ (Motion Control) are required (Jasperneite, 2005).

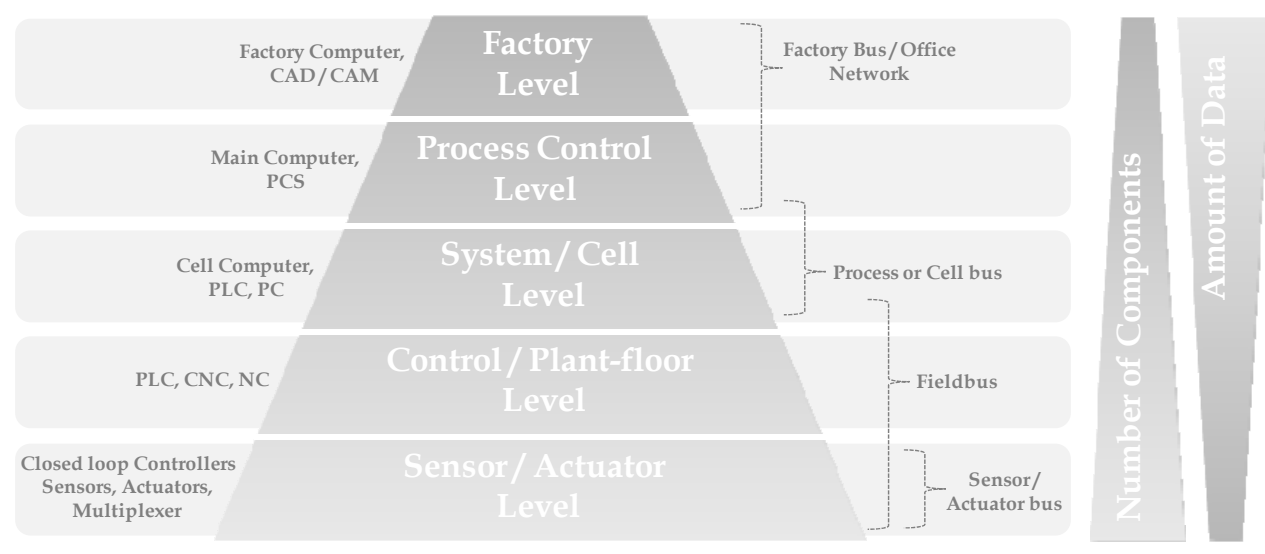

Fig. 1. The hierarchical automation pyramid.

The transition from control level to the sensor/actuator level is fluent. At this level sensors, actuators, drives, and manufacturing robots are equipped. The number of I/O-points is very high and densities of $2 / \mathrm{m}^{3}$ to $10 / \mathrm{m}^{3}$ can be expected in typical production plants (Scheible et al., 2007). The major part consists of sensors like proximity switches. The connection of sensors and actuators to PLCs can be realised in different ways. A differentiation is made between parallel and serial wiring. The classical parallel wiring uses analogue or digital (remote) I/O-Terminals to connect the sensors and actuators with the field bus system. New concepts, like IO-Link (IO-Link, 2009), use a 2/3-wire-standard-parallel-wiring of the sensors and actuators, as well. However, on the basis of a master/slave protocol, additional information, like diagnosis or parameters for configuration, can be exchanged with a PLC besides the process data. IO-Link specifies data rates of at least $4.8 \mathrm{kBaud}$ and $38.4 \mathrm{kBaud}$. Corresponding to the amount of process data, cycle times of $2 \mathrm{~ms}$ can be achieved at data rates of $38.4 \mathrm{kBaud}$. The serial wiring is usually realised by means of a sub bus system, which serially interconnects the sensors and actuators. The most representative technology is the AS-Interface (AS-Interface, 2009). At a maximal amount of 31 (62) devices, the cycle times are $<5 \mathrm{~ms}(10 \mathrm{~ms})$. Because of the high requirements concerning latency, drives and robots are interfaced directly to the field bus via appropriate application profiles or special drive buses, i.e. Sercos.

The application of wireless technologies is useful on the control as well as the sensor/actuator level. However, besides the stringent requirements concerning latency, determinism, reliability, and data integrity, the requirements with respect to costs, energy consumption, distances, bandwidth, node density, and network topologies differ.

Depending on the application, the requirements with respect to costs, energy consumption and node density are of minor importance on the control level. Because of the wide spreading of PLCs and decentralised peripherals, the ranges between devices may vary from a few meters up to some hundreds of meters. The process data of a whole plant is exchanged via the field bus system. In this conjunction the amount of process data of decentralised periph- 
erals may vary between $<10$ Bytes...> 100 Bytes. Hence, the bandwidth and data rates are of major importance. The size of the actual data packets depends on the structure of the field bus system and whether it uses multi-slave or single-slave frames. The network topologies for wireless solutions range from simple cable replacement point-to-point and point-tomultipoint connections up to cellular networks with roaming capabilities (production lines, automated guided vehicles).

Because of the high quantities of devices, the costs for acquisition, installation, commissioning, and operation are of major importance on the sensor/actuator level. The sphere of action is restricted to small production cells $\left(10 \mathrm{~m}^{3} \ldots 100 \mathrm{~m}^{3}\right)$ with high node densities. The amount of process data of a single sensor or actuator typically ranges from 1 Byte...10 Bytes. Hence, lower data rates and bandwidth are sufficient. In its simplest form, wireless solutions operate as cable replacements in point-to-point topology, as well. However, the development is focused on high speed wireless sensor/actuator networks (WSANs), supporting large numbers of devices. These networks are usually arranged in star topology and consist of wireless sensors/actuators, wireless I/O-concentrators, and a master base station, which acts as the interface to a super ordinate control system. Due to the increasing latencies, multihop topologies are currently not considered for WSANs in factory automation.

\section{Industrial Wireless Communication Channels}

Communication systems have to comply with the stringent requirements concerning reliability, availability, and determinism in order to serve automation applications. In contrast to that, the quality of a wireless transmission channel experiences random time and frequency variant fluctuations. Hence, the development of wireless communication systems, for the extreme time critical area of factory automation, is a big challenge.

Industrial environments are often characterised by a high degree of metallic surfaces and time-varying influences. Besides the movement of the radio systems itself the movements of materials/tools, rotating machines and persons are responsible for this time variant properties. In principle industrial radio channels are akin to mobile radio channels. Thus, most phenomena of industrial radio channels comply with the ones of mobile radio channels. The occurring physical phenomena of transmitted electromagnetic (EM) waves are illustrated in figure 2:

- Reflexions occur, when EM-waves encounter reflecting objects, whose dimensions are much larger than the wavelength.

- Scattering appears, either when the dimensions of the encountered object are much smaller than the wavelength of the EM-wave, or when the surface structure is classified very rough in comparison to the wavelength.

- Diffraction occurs when EM-waves encounter sharp edges.

- Shadowing is caused by obstacles, which completely block the propagation paths of EM-waves.

- Doppler effects arise, either when there is a relative movement between transmitter and receiver, or a mobile obstacle in the propagation field reflects, scatters, diffracts, or shadows the EM-wave. 


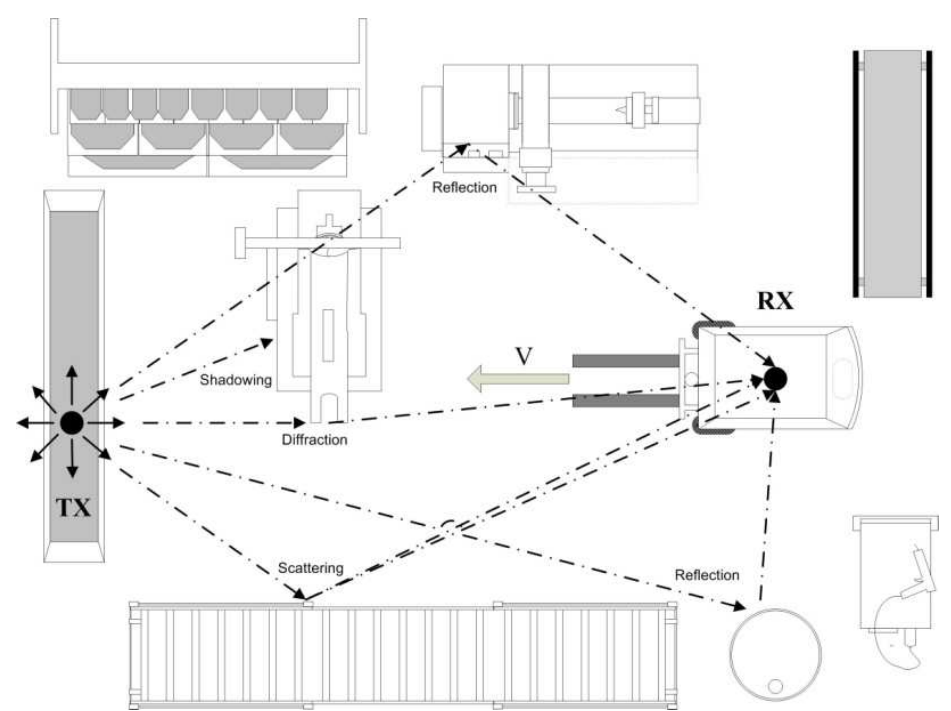

Fig. 2. The classical propagation of electromagnetic waves in a typical industrial environment.

Because of these wave phenomena a received signal is a composition of different attenuated and phase shifted versions of the original transmitted signal. Depending on the phase of these versions, a constructive or destructive overlapping occurs at the receiver. This effect is called multipath scattering. The absence of a direct non reflected version of the transmitted signal is typical for industrial radio channels. Does a relative proper motion between the transmitter and receiver take additionally place, or does the environment change due to rotating machines or forklift trucks, a shift in frequency based on the doppler effect influences the transmitted signal. Simultaneously, the path of the signal versions change, resulting in a new form of the received signal. Hence, the transmission behaviour of such a radio channel is time-variant and the signal power experiences high fluctuations.

\subsection{Large Scale Fading}

The large scale fading results from widespread movements. It depicts the mean signal power over spatial areas of about 10 wavelengths $\lambda$. Consequently, the local mean values of the propagation losses (path loss), which depend on the environment (shadowing, reflexion, diffraction, scattering), are characterised. In this conjunction the log-distance path loss model (Rappaport, 2002) is often used to describe path losses. The model states, that the mean received power $P_{r}$ decreases logarithmical with the distance $d$ between transmitter and receiver, following $P_{r} \propto P_{0}\left(d_{0} / d\right)^{\gamma}$. $d_{0}$ is a reference distance near the transmitter, where the transmit power $P_{0}$ is measured with respect to the far-field characteristics of the transmit antenna. The degree of signal attenuation is expressed by the path loss exponent $\gamma$. A detailed overview of the values of $\gamma$ is given in (Rappaport, 2002). In buildings $\gamma$ may vary very much. At frequencies of $400 \mathrm{MHz} . .4 \mathrm{GHz} \gamma$ can take values of $\gamma=\{2, \ldots, 6\}$ (Hashemi, 1993). In analysis of Rappaport (Rappaport, 2002; Rappaport \& Mcgillem, 1989, Rappaport, 
1989a), performed in five different factory environments, mean values of $\gamma=\{1.7, \ldots, 3\}$ were measured.

\subsection{Small Scale Fading}

Small scale fading characterises the fast fluctuations of radio channels over short distances (fraction $\lambda$ ). Primarily, these fast fluctuations of the channel are caused by doppler effects and multipath scattering. If, for example, a narrow band carrier signal is transmitted, several randomly organised signal copies arrive at the receiving antenna via different paths. For every location in a propagation environment, the received signal is the sum of all signal versions. If the signal versions, which arrive at the receiver, are uncorrelated in phase, the angles of arrival uniformly distributed, and the signal delay of each path much lower than the alteration speed of the radio channel, then the behaviour of attenuation can be described by two complex gaussian processes with mean values of $\mu=\mu_{R}+j \cdot \mu_{I}$. If there is no direct line of sight (NLOS) between transmitter and receiver, the mean value is $\mu=0$. In this case the probability distribution of the absolute amplitude values corresponds to the rayleigh distribution. If there is a direct line of sight (LOS), the mean value $\mu$ takes the amplitude value of the signal version, transmitted over the direct path $\mu=A_{\text {LOS }}$. The absolute amplitude values of these channels correspond to the rice distribution. Figure 3 shows a classical course of the absolute amplitude values of a rayleigh fading channel. The deep fades of up to $40 \mathrm{~dB}$ are characteristic. Analysis in industrial environments (Rappaport \& Mcgillem, 1989) showed a dynamic range of $20 \mathrm{~dB}$ in signal power, for stationary transmitters and receivers. When the receiver was moved with a velocity of $v=0.3 \mathrm{~m} / \mathrm{s}$, the dynamic range of the received signal increased to $30 \mathrm{~dB} \ldots 40 \mathrm{~dB}$. If a channel experiences such a deep fade, several channel errors occur, whose positions show a strong statistical dependence (Paetzold, 1999). The occurrence of channel errors temporarily appears in complex blocks.

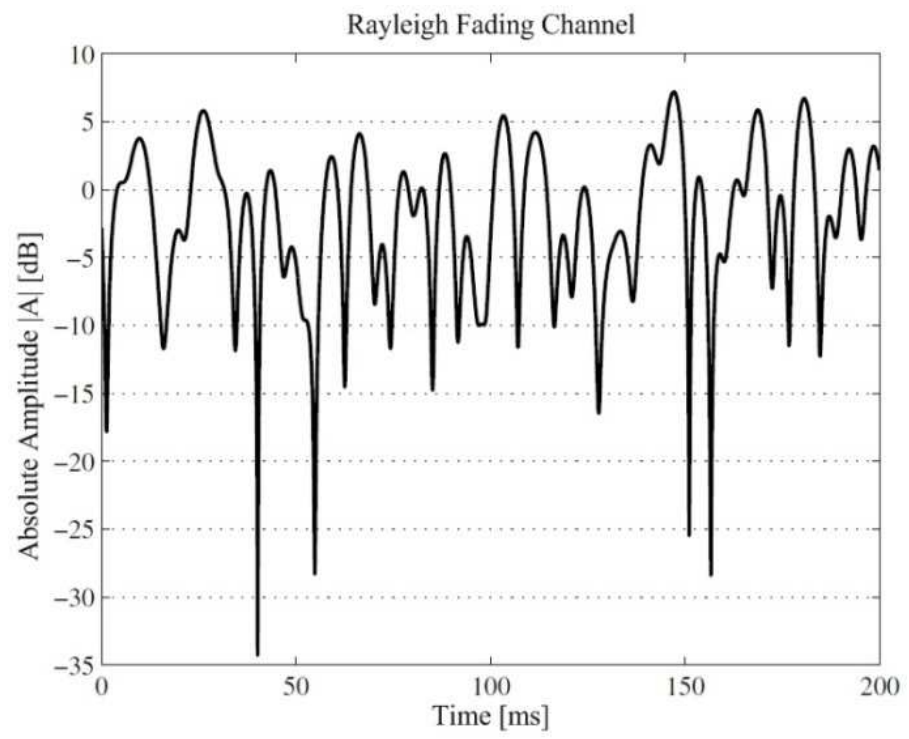

Fig. 3. The course of amplitudes of a rayleigh fading channel. 
Since the rayleigh and the rice models are derived on the assumption of a non modulated carrier signal, their application is restricted to narrow band signals.

In order to completely characterise a radio channel with respect to the domains of time and frequency, the time variant impulse response $\underline{h}(\tau, t)$ is an appropriate measure. On the supposition of a wide sense stationary uncorrelated scattering (WSSUS) channel, the following characteristics can be approximated on the basis of Fourier transformations of $\underline{h}(\tau, t)$ and the computation of first and second order statistics (Bello, 1963):

Delay spread:

The delay spread $\tau_{r m s}$ describes the mean spread in time of transmitted $\delta$-impulse. Scientific studies showed a delay spread of $\tau_{r m s}=20, \ldots, 30 \mathrm{~ns}$ at frequencies of $1.3 \mathrm{GHz}$ in industrial environments (Hashemi, 1993; Rappaport, 1989b). In this conjunction the works of Haehniche et al. (Haehniche et al., 2000; Haehniche, 2001) are of great practical interest. The delay spread for the $2.45 \mathrm{GHz}$ ISM frequency band was analysed in different industrial environments. A mean value of $72 \mathrm{~ns}$ and a maximal value of $121 \mathrm{~ns}$ were measured. Hoeing et al. (Hoeing et al., 2006) analysed the delay spread in a production cell with several scattering obstacles. The transmission distance was $3 \mathrm{~m}$ with LOS between transmitter and receiver. Within the propagation area of interest, fast cyclic movements of machines took place. Under these conditions a delay spread of $\tau_{r m s}=79 n s$ was measured, which corresponds to a path difference of about $23.7 \mathrm{~m}$ in length.

\section{Coherence bandwidth:}

Within a frequency area of $\Delta f$, which is smaller than the coherence bandwidth $B_{C}$, the course of ampitudes is expected to be constant. Between the delay spread and the coherence bandwidth the approximation $\tau_{r m s} \approx B_{C}^{-1}$ is valid. Haehniche et al. analysed the coherence bandwidth in different industrial environments, as well. Mean values of the coherence bandwidth $B_{C}=5.7 \mathrm{MHz}$ were measured for the $2.45 \mathrm{GHz}$ frequency band. In (Scheible, 2007) a coherence bandwidth of up to $10 \mathrm{MHz}$ is reported for this frequency range.

\section{Coherence time:}

The coherence time $T_{C}$ is a measure for a radio channels alteration speed.

\section{Doppler spread:}

The doppler spread $D_{S}$ describes the mean frequency spread of a tranmitted narrow band carrier signal. Between the coherence time and the doppler spread the approximation $T_{C} \approx D_{S}^{-1}$ is valid. The impact of the doppler spread in industrial radio channels may be enorm. Fast moving or rotating machines may induce high values of the doppler spread. Hoeing et al. have measured values for $D_{S}$ of up to $400 \mathrm{~Hz}$.

On the basis of the presented characteristics, the small scale fading can be further classified with respect to the variance in time and frequency of a radio channel. If the signal bandwidth is much smaller than the coherence bandwidth $B_{S} \ll B_{C}$, and the delay spread much smaller than the symbol duration $\tau_{r m s} \ll T_{S}$, the radio channel is characterised as flat fading (non frequency selective). Flat fading channels are often referred to as narrow band channels. If the signal bandwidth is larger than the coherence bandwidth $B_{S} \gg B_{C}$, the channel is frequency selective. In this case the delay $\tau$ of single paths is larger than the symbol duration $T_{S}$, what might induce intersymbol interferences (ISI) at the receiver. The time selectivity of a radio channel may either be described on the basis of the coherence time $T_{C}$ or the doppler spread $D_{S}$. If the symbol duration is much samller than the coherence time $T_{S} \ll T_{C}$, the form of the transmitted symbol is not altered by the radio channel. These channels are referred as 
slow fading (non time selective). The opposite is a time selective radio channel referred to as fast fading.

For a more detailed description of industrial radio channels the authors refer to (Vedral, 2007).

\subsection{Performance-Enhancing Strategies}

In order to comply with the challenging requirements of automation in the face of the depicted fluctuations of industrial radio channels, several performance enhancing strategies can be applied. It is obvious, that these methods are most effective, when implemented in the PHY or MAC layers. However, with the given architectures of available transceivers it is often necessary and only possible to implement appropriate protocols on application layer (Pellegrini et al., 2006).

Classical methods to improve the performance of radio channels are error detecting (retransmissions) or error correcting codes (Liu et al., 1997; Haccoun \& Pierre, 1996; Biglieri, 2005), which add further redundancy to the transmitted data. Since these methods are typically applied to a single channel, their effectiveness mostly depends on the small scale properties of the channel. Deep fades induce dense blocks of errors, which can be hardly corrected by error correcting codes. The success of a retransmitted signal depends on the duration of these deep fading (coherence time). A way to overcome these problems is the utilisation of diversity techniques. In general diversity describes the transmission of information over different channels. The achievable gain depends on the statistical independence of each transmission channel. With an increasing number of independent transmission channels the probability increases, that at least one channel is in a good state, and the transmitted signal can be decoded at the receiver. If the error generating processes are completely uncorrelated, the theoretical minimal error probability is $P_{r}=P_{e}^{n}$ for $\mathrm{n}$ transmission channels. Diversity techniques can be applied in the domains of time, frequency, space and angle. Since time diversity implies an increasing latency, its operation in time critical applications is not suitable. However, by applying spatial or frequency diversity, significant gains at reasonable costs can be achieved.

Spatial diversity may be applied in different forms. A classification is made for single-user and multi-user approaches. In the case of single-user, there is only one transmitter and one receiver, with at least one of which having multiple antennas. In (Diggavi, 2004) it is proven, that the achievable capacity nearly linearly increases with $N \rightarrow \infty$, if both transmitter and receiver are equipped with the same number of antennas $N$. In its simplest form, multiple antennas are used at the receiver (SIMO). The single signal versions are combined at the receiver in order to produce the received signal. Well known combining techniques are switched combining, equal gain combining or maximum ratio combining (Goldsmith, 2005). The achievable diversity gain thereby depends on the statistical independence of the received signals. On the assumption of a rayleigh fading channel the normalised correlation coefficients $\rho(\zeta)$ of two envelopes can be expressed as a function of antenna separation (Clarke, 1969) $\rho(\zeta)=J_{0}^{2} \cdot(2 \pi \zeta)$. $\zeta$ represents the seperation of two vertical monopole antennas in wavelengths and $J_{0}$ is the Bessel function of first kind and zero order (Zeppernick \& Wysocki, 1999). In (Vedral et al., 2007) practical measurements, in order to evaluate digital diversity techniques, were performed, based on a multi-transceiver platform, operating in the $2.45 \mathrm{GHz}$ frequency band. By utilising three receiving antennas at a separation of $4.69 \mathrm{~cm}$ a diversity gain of $3.5 \mathrm{~dB}$ could be realised in an industrial environment. Bit error 
rates (BER) could be reduced by half an order of magnitude compared to a single branch. The packet error rate (PER) could even be reduced by more than one order of magnitude. Based on more complex MIMO approaches (Boelcskei, 2006; Paulraj et al., 2004), i.e. applied in the upcoming standard IEEE 802.11n, performance gains can be further increased. The capabilities of multi-user approaches, i.e. relaying (Lanemann et al., 2004; Kramer et al., 2005), for industrial applications has been demonstrated in (Willig, 2008).

A second form of diversity is the transmission of Information over multiple frequencies. The achievable diversity gains depend on the statistical independence of the single transmission channels, as well. To obtain statistical independence between two channels their frequency separation should at least be larger than the actual coherence bandwidth. Following (Clarke, $1969)$, the normalised correlation coefficient $\rho(\Delta f)$ of two envolpes can be expressed as a function of frequency seperation $\rho(\Delta f)=(1+(2 \pi T \Delta f))^{-1 / 2}$. Thereby $\Delta f$ describes the seperation of the two frequencies and $T$ is the maximal delay spread of a current environment. In narrow band systems frequency diversity is often combined with time diversity in the form of "frequency hopping spread spectrum" (FHSS). In wide band systems, which use "orthogonal frequency division multiplex" (OFDM), frequency diversity is often applied on the basis of channel coding combined with interleaving in the frequency domain. In (Todd et al., 1992; Corazza et al., 1996) the performance of frequency diversity at frequencies of $1.75 \mathrm{GHz} . .1 .8 \mathrm{GHz}$ has been evaluated in typical office buildings. At an availability of $99 \%$, the achieved diversity gains varied between $5 \mathrm{~dB}$...9.6 dB for frequency separations larger than $5 \mathrm{MHz}$.

Having in mind the limitation of bandwidth and consumption of energy, spatial diversity is the more attractive strategy. However, frequency diversity is also considered a suitable instrument to compensate deep fading. Although it is proven, that optimum combining, using spatial diversity, may increase the signal to noise plus interference ration (SINR) in order to mitigate co-channel interferences (Winters, 1984), the application of frequency diversity is more effective and less complex.

\section{Current Wireless Base Technologies and its Utilisation in Factory Automation}

As already mentioned, most of the industrial wireless solutions use the unlicensed $2.45 \mathrm{GHz}$ ISM frequency band. This section gives an overview of the regulation and the most important technologies operating in this frequency range.

\subsection{Regulation for the $2.4 \mathrm{GHz}$ ISM Frequency Band}

Within the scope of the regulation 5.138 and 5.150 of the international telecommunication union, radiocommunication sector (ITU-R), besides others, the frequency range from $2.4 \mathrm{GHz}$ to $2.5 \mathrm{GHz}$ is enabled for industrial, scientific, and medical (ISM) applications. The European norm EN 300328 (ETSI 2006) regulates the frequency range from $2.4 \mathrm{GHz}$ to $2.4835 \mathrm{GHz}$ for general utilisation in Europe. The maximal EIRP transmit power is limited to $100 \mathrm{~mW}$. For devices, that do not use the modulation of "frequency hopping spread spectrum" (FHSS), the maximal spectral EIRP power density is further limited to $10 \mathrm{~mW} / \mathrm{MHz}$. There are no restrictions concerning the duty cycle of the radios. Depending on the application domain and the country, transmit powers above $10 \mathrm{~mW}$ have to be registered. In gen- 
eral, there are country specific limitations to the utilisation of the $2.45 \mathrm{GHz}$ ISM band (i.e. Spain and France).

In North America, the utilisation of unlicensed frequency bands is ruled by the Federal Communications Commission (FCC 2007) in the document CFR 47, Part 15. The maximal transmit power for the $2.45 \mathrm{GHz}$ band is limited to $1 \mathrm{~W}$ for systems using FHSS over more than 75 frequency channels. For systems with less than 75 channels, the maximal transmit power is limited to $125 \mathrm{~mW}$. In addition to that, a spectral power density of $8 \mathrm{dBm} / 3 \mathrm{kHz}$ must not be exceeded.

\subsection{Wireless Local Area Networks - IEEE 802.11}

The most popular radio technologies operating within the $2.45 \mathrm{GHz}$ band are compliant to the standards of IEEE $802.11 \mathrm{~b}$ and IEEE $802.11 \mathrm{~g}$. Both standards specify 13 channels with spacing of $5 \mathrm{MHz}$ for Europe and 11 for North America.

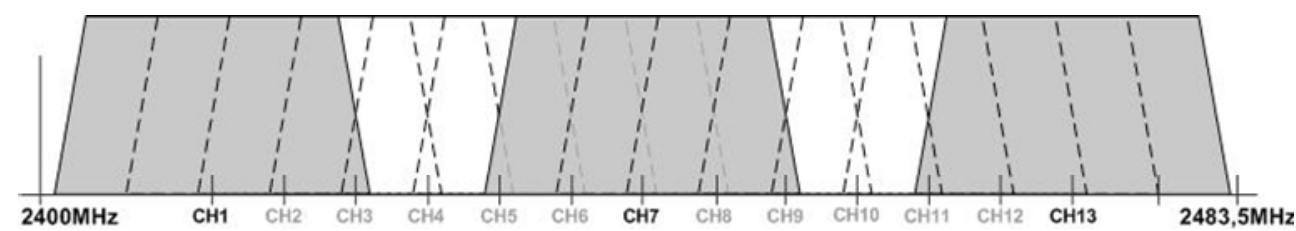

Fig. 4. IEEE 802.11 defines 13 channels for Europe and 14 Channels for North America.

With a transmit bandwidth of about $20 \mathrm{MHz}$, three non overlapping channels with a spacing of $30 \mathrm{MHz}$ are available. The maximal transmit power is limited to $100 \mathrm{~mW}$.

IEEE $802.11 \mathrm{~b}$ supports data rates of $1 \mathrm{Mbps...11} \mathrm{Mbps.} \mathrm{According} \mathrm{to} \mathrm{the} \mathrm{selected} \mathrm{data} \mathrm{rates,}$ the modulations of "differential binary phase shift keying" (DBPSK), "differential quadrature phase shift keying“ (DQPSK) or, „,complementary code keying" (CCK) are used. "Direct sequence spread spectrum" (DSSS) is used as a spreading technique. The amendment of IEEE $802.11 \mathrm{~g}$ is an extension and supports data rates of up to $54 \mathrm{Mbps}$ by introducing "orthogonal frequency division multiplex" (OFDM) with 52 sub-carriers as a spreading technique. These sub-carriers are either modulated using "binary phase shift keying“ (BPSK), "quadrature phase shift keying (QPSK), "16- or 64-quadrature amplitude modulation“ (16QAM, 64-QAM) depending on the selected data rates. Furthermore this standard supports forward error correction (FEC) with coding rates of $1 / 2,2 / 3$, or 3/4. As the channel access method, both standards use "carrier sense multiple access/collision avoidance", which is based on a "clear channel assessment" (CCA) module. Prior to any transmission, the CCA module validates the occupation of the medium. If the medium is classified "busy", the transmit operation is interrupted for a pseudo random period of time and the channel is validated again. A prioritised medium access, comprising eight priority levels, was introduced by the extension of IEEE 802.11e. In order to classify the medium, three modes are specified and one of them must at least be supported. In mode 1 the medium is considered busy, as soon as the detected energy is above a predefined threshold. In mode 2 the medium is considered busy, if an IEEE 802.11 modulated signal is detected. In mode 3 the medium is considered busy, if an IEEE 802.11 modulated signal is detected and its energy is above a predefined threshold. In general, the end-user has no access to the configuration of the CCA mode. 
In automation applications IEEE 802.11 is recommended by the PROFIBUS \& PROFINET International (PI) as a wireless communication system for connecting PLCs and decentralised peripherals. With adapted IEEE 802.11 systems, PROFINET-I/O communications with update times of up to $8 \mathrm{~ms}$ can be served. Common use cases are forklift trucks and automated guided vehicles. In mobile scenarios the transition from one cell to another (roaming) is extremely critical. Currently, roaming times of $<50 \%$ can be realised.

The next Amendment of the task group IEEE 802.11n is shortly before being published. This standard specifies either channels with $20 \mathrm{MHz}$ bandwidth and 56 OFDM sub-carriers and channels with $40 \mathrm{MHz}$ bandwidth and 112 sub-carriers within the frequency bands of $2.45 \mathrm{GHz}$ and $5 \mathrm{GHz}$. By applying performance enhancing techniques like "MIMO", "Channel Bonding", "Frame Aggregation", "Spatial Multiplexing", and "Beam forming", data rates of $300 \mathrm{Mbps}$ and beyond can be achieved. At the moment the draft standard, revision 8 , is available (LAN/MAN Standards Committee of the IEEE Computer Society, 2008). The release of the final standard is expected in late 2009. Similar to the standards IEEE 802.11b and IEEE $802.11 \mathrm{~g}$ a fast market penetration can be expected for the standard IEEE 802.11n, as well.

\subsection{Bluetooth - IEEE 802.15.1}

The latest specification of Bluetooth version 3.0 (Bluetooth Special Interest Group - SIG, 2009) was published in 2009. The PHY and MAC layer of the Bluetooth version 1.1 are published as the standard IEEE 802.15.1, as well. In its classical form 79 channels, with a spacing of $1 \mathrm{MHz}$, are specified in the range of $2.402 \mathrm{GHz} \ldots .2 .480 \mathrm{GHz}$. The radio signals are modulated using "Gaussian frequency shift keying" (GFSK, $1 \mathrm{Mbps}$ ), " $\Pi / 4$ differential quaternary phase shift keying" (П/4-DQPSK, 2 Mbps), or "8-ary differential encoded phase shift keying" (8DPSK, 3 Mbps). Bluetooth uses "Time Division Multiple Access" (TDMA) as the channel access method and FHSS for spreading. Three device classes with transmit powers of $1 \mathrm{~mW}, 2.5 \mathrm{~mW}$ and $100 \mathrm{~mW}$ are defined.

Bluetooth networks, called piconets, are formed in star topology. A piconet consists of a master and up to seven active slaves. In order to communicate, timeslots with a length of $625 \mu$ s are predefined. The specification defines synchronous connections (SCO) for the transmission of i.e. speech and asynchronous connections (ACL) for data transmission. Depending on the type, data packets occupy one to five timeslots and use "automated repeat requests" (ARQ) or FEC as channel coding. In each timeslot, or at leas after the transmission of a data packet, a change in frequency is performed respectively.

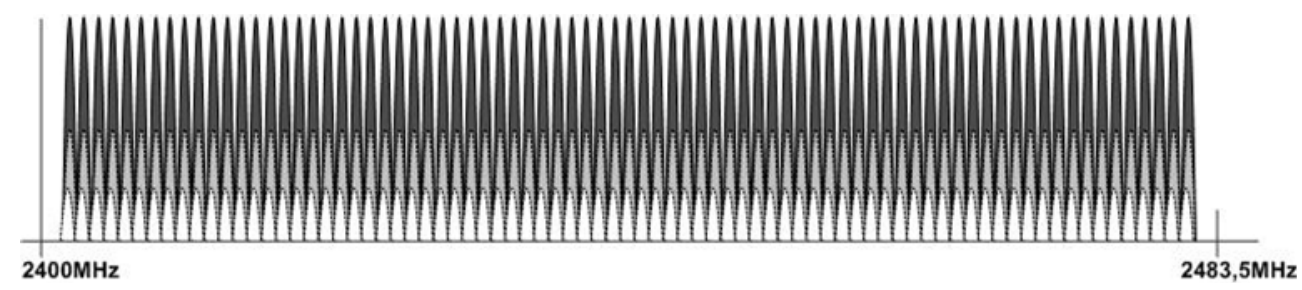

Fig. 5. IEEE 802.15.1 defines 79 Channels within the 2.45 GHz ISM Band.

In avoidance of coexistence problems, the standard supports an "adaptive power control" (APC) and "adaptive frequency hopping" (AFH). When using AFH, frequency channels 
occupied by foreign radios are detected and excluded from the hopping scheme. With common Bluetooth transceiver chips a channel is classified busy, when the occupation is higher than $15 \%$. The adaption of the hopping scheme depends on the implementation and may take up to several seconds. In addition to the adaptive channel classification, frequency channels can be excluded of the hopping scheme manually, in order to avoid frequencies known to be in use by other radios. At least 20 channels have to be used. By doing so, a frequency separation to two coexisting IEEE 802.11 radios can be administered. Solely, the connection setup uses all frequencies. However, some vendors developed standard compliant solutions, which prevent interferences during the connection setup.

Bluetooth is applicable at control as well as sensor/actuator level. With respect to ABBs "Wireless interface for sensors and actuators" (WISA), the PROFIBUS \& PROFINET International (PI) actually considers the PHY layer of Bluetooth as the basis for "Wireless Sensor/Actor Networks" (WSANs). A standard shall be published in 2010. A WISA network consists of a base station and up to 120 wireless I/O-concentrators and sensors/actuators in a star topology. The base station acts as the network coordinator and gateway to a super ordinate control system. The I/O-concentrators and sensors/actuators use IEEE 802.15.1 standard compliant transceivers. The base station consists of a special multi-transceiver architecture and thus able to serve multiple devices in parallel. The update time of 120 sensors is typically below $20 \mathrm{~ms}$.

In version 3.0 of Bluetooth, the support of IEEE 802.11 as an "Alternate MAC PHY" (AMP) is introduced. In addition to that the "Bluetooth Low Energy" specification is to be published in late 2009. First transceivers for both technologies shall be available in 2010.

\subsection{IEEE 802.15.4}

The standard IEEE 802.15.4 specifies 16 channels with a separation of $5 \mathrm{MHz}$ for the $2.45 \mathrm{GHz}$ ISM band. With DSSS as spreading and "offset quadrature phase shift keying" (OQPSK) as modulation, data rates of $250 \mathrm{kbps}$ are supported. The standard limits the transmit power to $1 \mathrm{~mW}$. However, the regulations allow the operation at transmit powers of up to $10 \mathrm{~mW}$.

As channel access method CSMA/CA corresponding to IEEE 802.11 is utilised. Optionally, the standard supports a synchronised data communication in superframes of durations from $15 \mathrm{~ms}$ to $246 \mathrm{~s}$. Each superframe consists of a "contention access period" (CAP) and a "contention free period" (CFP). During the CAP, devices willing to transmit, concurrently access the medium via CSMA/CA. The CFP consists of guaranteed timeslots and gives exclusive access to medium for higher prioritised transmissions. The standard was designed for low power industrial "wireless personal area networks" with low data rates.

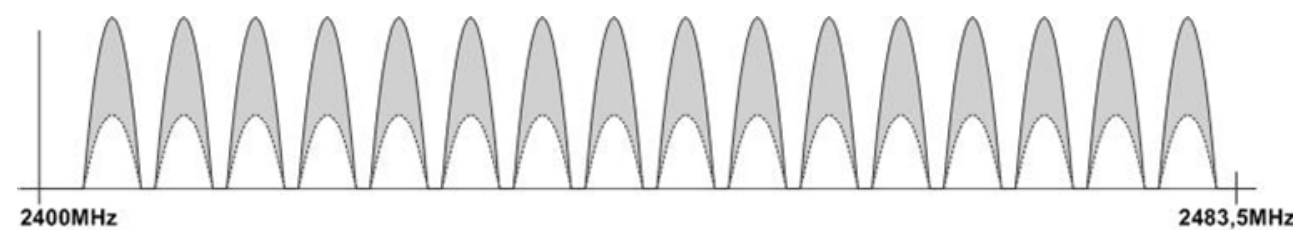

Fig. 6. IEEE 802.15.4 defines 16 Channels within the 2.45 GHz ISM Band. 
The technology is wide spread in combination with the higher layers specified by ZigBee. ZigBee supports the operation of large multihop networks and addresses domains like home- and building automation, smart metering, and health care.

Within the scope of the HART 7 specifications, the first wireless standard for process automation, WirelessHART, was published in 2007. WirelessHART is based on the PHY layer of IEEE 802.15.4 and uses the "Time Synchronized Mesh Protocol" (TSMP) for channel access. In order to improve reliability, it is designed to support large multihop networks in full mesh topologies with a high degree of redundant paths. In avoidance of coexistence problems the standard changes frequencies at a rate of $10 \mathrm{~ms}$. Optionally, a channel black list can be used to avoid frequencies currently in use. First products are successfully in use since late 2008.

At the moment "the International Society of Automation" (ISA) is shortly before publishing a second standard for the process automation, ISA 100.11a (ISA, 2009), based on the PHY layer of IEEE 802.15.4.

In the domain of factory automation a few proprietary solutions for the transmission of sensor data based on IEEE 802.15.4 are available.

Right now the task group of IEEE 802.15.4e is working on MAC layer extensions. In order to improve the support of time critical industrial applications, shorter transmit times, improved TDMA techniques and frequency hopping are evaluated. In the long run the extensions of IEEE 802.15.4e shall enable the standard to better support applications in factory automation.

\subsection{Coexistence in the $2.4 \mathrm{GHz}$ ISM Frequency Band}

With the fast pace growth of wireless solutions, operating in the $2.45 \mathrm{GHz}$ ISM band, in automation as well as the IT, the end-users demand for a good coexistence of the devices is getting obvious. In this respect a technologies coexistence properties depend on several parameters, like the transmit power, signal bandwidth, channel access methods, and dutycycle, which often are vendor specific.

In IEEE 802.15.2 (LAN/MAN Standards Committee of the IEEE Computer Society, 2003) coexistence is defined as "a systems ability to perform a task in a shared medium, while other systems perform their tasks, complying with the same or a different set of rules". In a shared medium the main source of error is caused by interferences. Interferences appear, when signals overlay in the domains of time, frequency, and space. For the domain of frequency the IEEE Unapproved Draft Std P1900.2/D2.22 (LAN/MAN Standards Committee of the IEEE Computer Society, 2007b) further subdivides interferences into "In-Band", consisting of "Co-Channel-" and "Adjacent Channel- Interference", and "Out of Band", consisting of "Band Edge-" und "Far out of Band Interference". The most common form of appearance are "Co-Channel" interferences, which occur, when more than one system operates on the same frequency. 


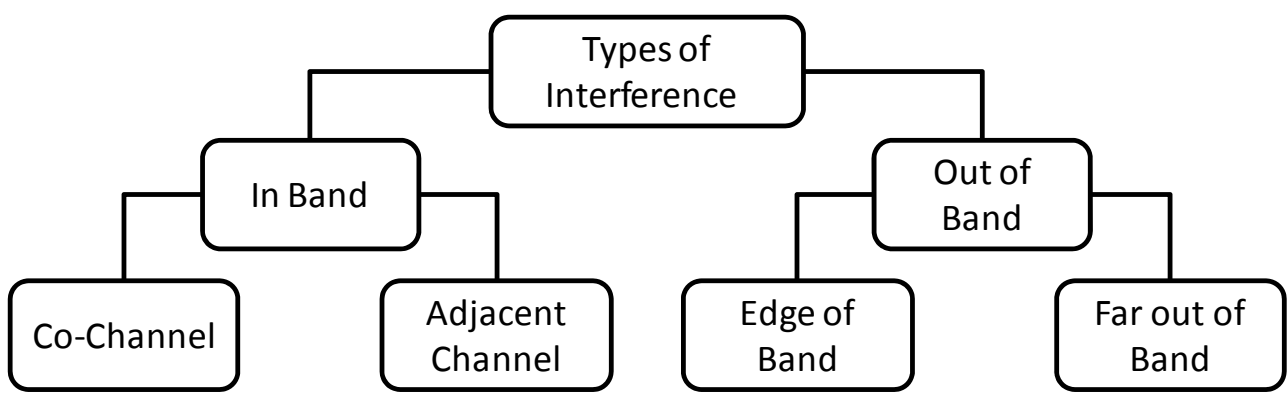

Fig. 7. Types of Interference defined by IEEE P1900.2/D2.22.

The domain of time is determined by the channel occupation in time, the duty cycle, of coexisting systems. The probability of signal interferences increases with the utilisation of the medium in time. The spatial domain is defined by the transmit power, the distance between the systems (Antennas), and the resulting "signal to interference plus noise ratio" (SINR). If the SINR is too low, a signal cannot be detected correctly at the receiver.

Besides these physical properties of interferences, channel access methods have a strong impact on the coexistence of radios. Typically, radio systems operating in the $2.45 \mathrm{GHz}$ ISM band use either TDMA, CSMA/CA, or a mixture of both as access methods. TDMA subdivides the medium into timeslots, which are reserved for exclusive access to the medium. That way, TDMA systems support a deterministic behaviour in time and a good coexistence within the same network. In order to avoid interferences to foreign networks, TDMA is often used in combination with FHSS, additionally allowing to black list frequencies already in use by other systems (i.e. Bluetooth). When using CSMA/CA, the state of the medium is validated before any transmission of data and only performed, if the medium is classified idle. The validation of the medium is either based on an energy threshold, the detection of a valid carrier, or a mixture of both. On the one hand CSMA/CA is able to avoid interferences within the same or foreign networks. On the other hand CSMA/CA is vulnerable to jamming attacks and some kind of unnecessary interferences. Depending on the implementation, the following types of interferences may occur, when using CSMA/CA:

- Type-1: A weak signal, that would not induce interferences at the receiver, is detected at the transmitter, causes the medium to be classified busy, and thus delays the transmission ("Exposed Terminal Problem").

- Type-2: Interferences caused by multiple radios that access the medium at the same time.

- Type-3: The source of interference is out of the detection range of the transmitter, but causes interferences at the receiver ("Hidden Terminal Problem").

There are several strategies to mitigate these interferences within the same network of operation (Tsertou \& Laurenson, 2008; Zhang et al., 2008). However, interferences with foreign networks may still appear.

How far interferences actually influence the coexistence properties of a system, always depends on the tasks to be performed. Usually, an underlying (wireless) communication system has a temporal reserve with respect to an application, in order to perform channel coding and retransmissions. If this reserve gets exhausted, the communication system cannot longer serve the application. It is obvious, that with increasing temporal requirements of an 
application, the reserve of the communication system decreases and interferences result in application errors faster. Analytical as well as practical studies about the coexistence within the $2.45 \mathrm{GHz}$ ISM band have been subject to several publications. For detailed information on this topic it is referred to (Arumugm et al., 2003; Chiasserini \& Rao, 2003; Howitt \& Gutierrez, 2003).

The previous descriptions stated the richness of technologies and applications operating in the $2.45 \mathrm{GHz}$ ISM band. Thus, a coexisting operation of different wireless solutions is hardly avoidable. But it is very demanding to consider all parameter of relevance for the different domains of applications, when determining the properties of coexistence of radio technologies. In addition to that, comprehensive studies on the coexistence of new technologies, like IEEE 802.11n, WirelessHART, ISA 100.11a, and Bluetooth Low Energy have not been performed, yet.

\begin{tabular}{|c|c|c|c|}
\hline Category & Class & Application & Description \\
\hline Safety & 0 & Emergency action & (always critical) \\
\hline \multirow{3}{*}{ Control } & 1 & Closed loop regulatory control & (often critical) \\
\cline { 2 - 4 } & 2 & Closed loop supervisory control & (usually non-critical) \\
\cline { 2 - 4 } Monitoring & 3 & Open loop control & $\begin{array}{c}\text { Short-term operational conse- } \\
\text { quences (e.g. event-based } \\
\text { maintenance) }\end{array}$ \\
\cline { 2 - 4 } & 5 & Alerting & $\begin{array}{c}\text { No immediate operational } \\
\text { consequence (e.g., history } \\
\text { collection, sequence-of-events, } \\
\text { preventive maintenance) }\end{array}$ \\
\hline
\end{tabular}

Table 1. Application classes of ISA-SP100.

For that reason, a general process to establish a coexistence management for end user is described in (VDI, 2008). In relation to the application classes defined in (ISA, 2006), it is recommended to assign priorities to the different wireless solutions. The intensity for the frequency management shall be correlated to the assigned priority classes. The process comprises the whole plant location and shall include all persons responsible for planning, installing, and commissioning of wireless devices. Wireless applications either in automation, logistic, or IT have to be considered. The coexistence management is a cyclic process which comprises all stages of stock taking, planning, installation, commissioning, maintenance, operation, and documentation of wireless applications at a location. It is further recommended to involve qualified service providers and own personnel at early phases, in avoidance of malfunctions in the long run.

\section{Upcoming Wireless Base Technologies}

The development of wireless technologies and extended standards is fast pacing. Especially the progress with respect to ultra wideband (UWB) represents a great potential, to open up new domains of applications in factory automation. First efforts for a standardisation of UWB technologies were initiated by the IEEE 802.15 WPAN High Rate Alternative PHY Task Group 3a (TG3a), founded in 2001. The task groups aim was to develop a high speed 
UWB technology, supporting data rates of $>100 \mathrm{Mbps}$ at distances of $<10 \mathrm{~m}$. Unfortunately, the group was not able to reach a consensus between two approaches offered by the leading industrial consortiums of the "WiMedia Alliance" and the "UWB Forum" and hence, disbanded in 2006. However, the approach of the WiMedia Alliance was published as the standard ECMA-368 in 2006 and is available in version 3.0 (Ecma International, 2008) since 2008. The standard uses "Multiband OFDM" (MB-OFDM) as modulation and supports data rates of up to $480 \mathrm{Mbps}$ at distances of $<10 \mathrm{~m}$. MB-OFDM is the basis of "Certified Wireless USB" (CW-USB). The application as an "Alternate MAC PHY" (AMP) is evaluated by the Bluetooth SIG. First transceiver chips and products are available since 2007. In 2007 the IEEE 802.15 WPAN Low Rate Alternative PHY Task Group 4a (TG4a) (LAN/MAN Standards Committee of the IEEE Computer Society, 2007c) published the second UWB standard. IEEE 802.15.4a is a low data rate UWB technology supporting data rates of $0.1 \mathrm{Mbps} . .27 \mathrm{Mbps}$. It targets industrial sensor networks with real-time location capabilities. First transceiver chips will be available in 2010.

\subsection{Ultra Wideband}

In principle UWB is an old technology, whose origins come from military applications of the USA, more than 40 years ago. Whilst back then UWB was used as a tap-proof radio communication, nowadays the applications aim at high speed data transfers and real-time location systems. The first regulation for UWB devices, published by the FCC in 2002 (FCC, 2007), defines UWB as follows. The relative bandwidth has to be larger than $20 \%$ and the absolute bandwidth has to be at least $500 \mathrm{MHz}$ at a $10 \mathrm{~dB}$ cut-off frequency. The regulation gives no restrictions concerning signal forming and modulation. Because of the dense occupied frequency spectrum, UWB follows the approach of a parallel utilisation of the spectrum with a large bandwidth and a low spectral density power. In doing so, UWB appears as noise to coexisting narrow band technologies.

Classically, UWB is based on "Impulse Radio" (Nekoogar, 2005), which transmits information via impulses in the baseband without modulation. The UWB spectrum is generated due to extreme short durations ( $<1 n s)$ of these impulses. Hence, UWB has the following inherent characteristics :

- Low latency times, due to extreme short symbol durations, what additionally offers the possibilities for precise ranging.

- $\quad$ Robust against the effects caused by multipath scattering. Reflexion and scattering are frequency selective. Using a high bandwidth reduces the probability of deep fading.

- Energy efficiency, due to the low spectral density power.

- Data rates of up to several Gbps.

Especially the first characteristics prove the potential of UWB for industrial communication systems. A typical use case would be a cable replacement for high speed real-time Ethernet field buses. Further use cases are WSANs. First studies on this issue have been published in (Paselli et al., 2008). A general overview of the potential use cases of UWB in industrial applications is given in (Hancke \& Allen, 2006). 


\subsection{Regulation for Ultra Wideband}

Since UWB uses frequencies, which are already in use by licensed radio applications, the regulations are relatively restricted in order to avoid interferences to these applications. The first regulation was published by the FCC Part 15 Subpart F in 2002. The document defines seven classes of UWB devices. The classes of importance for factory automation are "Indoor UWB systems" and "Hand held UWB systems". "Indoor UWB systems" may only be used inside buildings and must have a fixed indoor infrastructure (i.e. power supply). "Hand held UWB systems" may operate indoor or outdoor and must not have a fixed infrastructure. The frequency ranges and maximal allowed transmit powers are depicted in table 2 .

\begin{tabular}{|l|l|l|}
\hline \multirow{2}{*}{ Frequency [MHz] } & Max. EIRP [dBm/MHz] \\
\cline { 2 - 3 } & Indoor UWB systems & Hand held UWB systems \\
\hline $960-1.610$ & -75.3 & -75.3 \\
\hline $1.610-1.990$ & -53.3 & -63.3 \\
\hline $1.990-3.100$ & -51.3 & -61.3 \\
\hline $3.100-10.600$ & -41.3 & -41.3 \\
\hline$<10.600$ & -51.3 & -61.3 \\
\hline
\end{tabular}

Table 2. Maximum EIRP spectral power densities for "Indoor UWB systems" and "Hand held systems" defined by FCC Part 15 Subpart F.

In further avoidance of interferences to GPS applications the maximal EIRP power density is limited to $-83.3 \mathrm{dBm} / \mathrm{kHz}$ for the frequency ranges of $1.164 \mathrm{GHz} \ldots 1.240 \mathrm{GHz}$ and $1.559 \mathrm{MHz} . .1 .610 \mathrm{MHz}$. Within a frequency spectrum of $50 \mathrm{MHz}$ the maximal power is limited to $0 \mathrm{dBm}$. It is obvious that the actual range of operation is between $3.1 \mathrm{GHz} . .10 .6 \mathrm{GHz}$.

The regulation for the frequency range from $3.1 \mathrm{GHz} . .10 .6 \mathrm{GHz}$ for harmonised utilisation of UWB systems in Europe was released in 2007 by the decision of the European commission (European Commission, 2007). The decision defines maximal EIRP power densities in $\mathrm{dBm} / \mathrm{MHz}$ and within a spectrum of $50 \mathrm{MHz}$ (comp. table 3). In addition to that, the decision differentiates between devices, which implement mitigation techniques in order to increase protection for radio Services. One mitigation technique is defined as "low duty cycle“ (LDC). Devices implementing LDC must have a duty cycle lower than $0.5 \%$ per hour and lower than $5 \%$ per second. Furthermore, a single transmit duration must not exceed $5 \mathrm{~ms}$. Another technique is "detect and avoid" (DAA). Devices implementing DAA shall observe the used frequency spectrum with respect to coexisting devices and must adapt their transmit behaviour to avoid interferences.

\begin{tabular}{|l|l|l|}
\hline Frequency $[\mathrm{GHz}]$ & $\begin{array}{l}\text { Max. EIRP Power Density } \\
(\mathbf{d B m} / \mathbf{M H z})\end{array}$ & $\begin{array}{l}\text { Max. EIRP Power Density } \\
(\mathbf{d B m} / \mathbf{5 0} \mathbf{~ M H z})\end{array}$ \\
\hline$<1.6$ & -90.0 & -50.0 \\
\hline $1.6-3.4$ & -85.0 & -45.0 \\
\hline $3.4-3.8$ & -85.0 & --45.0 \\
\hline $3.8-4.2$ & -70.0 & -30.0 \\
\hline
\end{tabular}




\begin{tabular}{|l|l|l|}
\hline $4.2-4.8$ & $-41.3(-70.0)$ & $-0.0(-30,0)^{1}$ \\
\hline $4.8-6.0$ & -70.0 & -30.0 \\
\hline $6.0-8.5$ & -41.3 & -0.0 \\
\hline $8.5-10.6$ & -65.0 & -25.0 \\
\hline$>10.6$ & -85.0 & -45.0 \\
\hline
\end{tabular}

Table 3. Maximum EIRP spectral power densities for Europe.

Table 2 shows, that the utilisation of frequencies below $6 \mathrm{GHz}$ will be restricted to devices, implementing mitigation techniques. How far real-time applications in factory automation can be served, regarding these restrictions, has to be investigated.

\section{Conclusion}

Industrial environments are highly demanding for the utilisation of wireless communication systems. However, on the basis of suitable adaptations and performance enhancing strategies several applications in factory automation can already be served by radio solutions. The current state of the art reliably supports update times of about $10 \mathrm{~ms}$ on application layer. First standards for the domain of factory automation based on the PHY layer of Bluetooth can be expected in 2010. Due to the huge deployment of wireless technologies, using the $2.45 \mathrm{GHz}$ ISM band, either in automation and IT, the problem of interferences, caused by coexisting devices, increases. In order to guarantee a reliable communication, even for time critical applications, a plant wide coexistence management is absolutely essential. By using other frequency ranges, the emerging UWB technologies give a great potential, to ease these coexistence problems. Furthermore they offer the possibility of addressing applications with temporal requirements of about $1 \mathrm{~ms}$ and below, because of their extreme short symbol durations. The research on UWB for industrial applications, especially factory automation, has just started. The upcoming years are going to reveal, whether UWB will enter into the domain of factory automation or not.

\section{References}

Arumugam, A.K.; Doufexi, A.; Nix, A. R.; Fletcher, P.N. (1003). An Investigation of the Coexistence of 802.11g WLAN and High Data Rate Bluetooth Enabled Consumer Electronic Devices in Indoor Home and Office Environments, IEEE Trans. on Consumer Electronics, vol. 49, no. 3, pp. 587-596, Aug. 2003.

AS-Interface (2009) (online). Website: http:/ / www.as-interface.net, visited on May 2009

Bello, P. A. (1963). Characterization of Randomly Time-Variant Linear Channels. In: IEEE Transactions on Communication Systems 11, pp. 360-393, Dec. 1963.

Biglieri, E. (2005). Coding for Wireless Channels. New York: Springer, 2005.

Bluetooth Special Interest Group - SIG (2009): Specification of the Bluetooth System, Version 3.0. Bluetooth Special Interested Group, 2009.

Boelcskei, H. (2006). Mimo-ofdm wireless systems: Basics, perspectives and challenges. IEEE Wireless Communications 13(4), pp. 31-37. 
Clarke, R. H.(1969). A statistical theory of mobile radio reception, The Bell System Technical Journal, vol. 47, pp. 957-1000, Aug. 1969.

Chiasserini, C.-F.; Rao, R. R. (2003). Coexistence mechanisms for interference mitigation in the 2.4-ghz ism band, IEEE Trans. on Wireless Communications, vol. 2, no. 5, pp. 964975, Sept. 2003.

Corazza, G.E.; Degli-Esposti, V.; Frullone, M.; Riva, G. (1996). A characterization of indoor space and frequency diversity by ray-tracing modeling, IEEE Journal on Selected Areas in Communications, Volume 14, Issue 3, Apr 1996 Page(s):411 - 419

Diggavi, S. N.; Al-Dhahir, N.; Stamoulis, A.; Calderbank, A. R. (2004). Great Expectations: The Value of Spatial Diversity in Wireless Networks, Proceedings of the IEEE, vol. 92, no. 2, pp. 219-270, Feb. 2004.

Ecma International (2008), Standard ECMA-368: High Rate Ultra Wideband PHY and MAC Standard, 3rd Edition.

ETSI (2006). EN 300 328, Electromagnetic compatibility and Radio spectrum Matters (ERM); Wideband transmission systems; Data transmission equipment operating in the 2,4 GHz ISM band and using wide band modulation techniques; Harmonized EN covering essential requirements under article 3.2 of the R\&TTE Directive", V1.7.1.

European Commission (2007). COMMISSION DECISION of 21 February 2007 on allowing the use of the radio spectrum for equipment using ultra-wideband technology in a harmonised manner in the Community, document number $C(2007)$ 522, 2007/131/EC.

Federal Communications Commission FCC (2007). 02 48A1 Revision of Part 15 of the Commission's Rules Regarding Ultra-Wideband Transmission Systems, February 2002, revision of 2007

Goldsmith, A. (2005). Wireless Communications, Cambridge University Press, 40 West 20th Street, NY 10011-4211, 2005.

Haccoun, D.; Pierre, S. (1996). Automatic repeat request," in The Communications Handbook, J. D. Gibson, Ed. Boca Raton, Florida: CRC Press / IEEE Press, 1996, pp. 181-198.

Haehniche, J. ; Rauchhaupt, L. (2000). Radio Communication in Automation Systems: the RFieldbus Approach. In: Proceedings of the IEEE Workshop on Factory Communication Systems (WFCS 2000), 2000, S. 319-326.

Haehniche, J. (2001). Radio based communication in automation - Overview of technologies (in german), Practical automation (in german), ATP 43 (2001), Jun., Nr. 6, S. 22-27.

Hancke, G.P.; Allen, B. (2006). Ultra wideband as an Industrial Wireless Solution, IEEE Pervasive Computing, Vol. 5, Issue 4, pp. 78 - 85, Oct.-Dec. 2006

HART Communication Foundation (2008), HART Field Communication Protocol Specifications, Revision 7.2, 2008

Hashemi, H. (1993). The Indoor Radio Propagation Channel. In: IEEE Transactions on Communications 81 (1993), Mai, Nr. 7, S. 943-968

Hoeing, M.; Helmig, K.; Meier, U. (2006): Analysis on the interference immunity and communication reliability of the Bluetooth technology using the example of an industrial sensor/actor network (in german), In: VDI Progress Reports (in german), Bd. 10, Nr. 772, 2006, S. 155-164

Howitt, I.; Gutierrez, J. A. (2003). IEEE 802.15.4 low rate - wireless personal area network coexistence issues, in Proc. Wireless Communications and Networking Conference 2003 (WCNC 2003), New Orleans, Louisiana, Mar. 2003, pp. 1481-1486. 
IEC (2007a), Geneva. Industrial communication networks - Fieldbus Specifications. IEC 61158 Ed. 4.0.

IEC (2007b), Geneva. Industrial communication networks - Profiles - Part 1: Fieldbus profiles. IEC 61784-1 Ed. 2.0.

IEC (2007c), Geneva. Industrial communication networks - Profiles - Part 2: Additional fieldbus profiles for real-time networks based on ISO/IEC 8802-3. IEC 61784-2 Ed. 1.0 .

IO-Link (2009) (online). Website: http:/ / www.io-link.com, visited on May 2009

ISA (2009) SP100.11a Working Group for Wireless Industrial Automation Networks: "Wireless systems for industrial automation: Process control and related applications"

ISA (2006) SP100.11, Call for Proposal, Wireless for Industrial Process Measurement and Control.

Kramer, G.; Gastpar, M.; Gupta, P. (2005). Cooperative strategies and capacity theorems for relay networks. IEEE Transactions on Information Theory 51(9), 3037-3063.

Konnex Association, Volume 3: System Specification," Version 1.3, 2006

LAN/MAN Standards Committee of the IEEE Computer Society (2003). IEEE Standard for Information technology - Telecommunications and information exchange between systems - Local and metropolitan area net-works - Specific requirements - Part 15.2: Coexistence of Wireless Personal Area Networks with Other Wireless Devices Operating in Unlicensed Frequency Bands.

LAN/MAN Standards Committee of the IEEE Computer Society (2005). IEEE Standard for Information technology - Telecommunications and information exchange between systems - Local and metropolitan area net-works - Specific requirements - Part 15.1: Wireless Medium Access Control (MAC) and Physical Layer (PHY) Specifications for Wireless Personal Area Networks (WPANs).

LAN/MAN Standards Committee of the IEEE Computer Society (2006). IEEE Standard for Information technology - Telecommunications and information exchange between systems - Local and metropolitan area net-works - Specific requirements - Part 15.4: Wireless Medium Access Control (MAC) and Physical Layer (PHY) Specifications for Low Rate Wireless Personal Area Networks (LR-WPANs). revision of 2006.

LAN/MAN Standards Committee of the IEEE Computer Society (2007a). Information technology - Telecommunications and Information Exchange between Systems - Local and Metropolitan Area Networks - Specific Requirements - Part 11: Wireless LAN Medium Access Control (MAC) and Physical Layer (PHY) Specifications.

LAN/MAN Standards Committee of the IEEE Computer Society (2007b). IEEE Standard for Information technology - Telecommunications and information exchange between systems - Local and metropolitan area net-works - Specific requirements - Part 19: Unapproved IEEE Draft Recommended Practice for Interference and Coexistence Analysis, IEEE Unapproved Draft Std P1900.2/D2.22.

LAN/MAN Standards Committee of the IEEE Computer Society (2007c). IEEE Standard for Information technology - Telecommunications and information exchange between systems - Local and metropolitan area networks - Specific requirements - Part 15.4a: Wireless Medium Access Control (MAC) and Physical Layer (PHY) Specification for Low-Rate Wireless Personal Area Networks (LR-WPANs), Amendment 1: Add Alternate PHY 
LAN/MAN Standards Committee of the IEEE Computer Society (2008). IEEE Standard for Information technology - Telecommunications and information exchange between systems - Local and metropolitan area networks - Specific requirements - Part 11n: Wireless LAN Medium Access Control (MAC) and Physical Layer (PHY) specifications. Amendment 4: Enhancements for Higher Throughput, IEEE Draft STANDARD, revision of 2008

Liu, H.; Ma, H.; Zarki, M. E.; Gupta, S. (1997). Error control schemes for networks: An overview, MONET - Mobile Networks and Applications, vol. 2, no. 2, pp. 167-182, 1997.

Laneman, J. N.; Tse; D.; Wornell, G. W. (2004). Cooperative diversity in wireless networks: Efficient protocols and outage behaviour. IEEE Transactions on Information Theory 50(12), 3062-3080.

Nekoogar, F. (2005). Ultra-Wideband Communications-Fundamentals and Applications, Prentice Hall Communications Engineering and Emerging Technologies Series. ISBN: 0-13146326-8, 2005

Paetzold, M. (1999). Mobile radio channels (in german), Wiesbaden : Vieweg Verlag, 1999

Paselli, M.; Petre, F.; Rousseaux, O.; Meynants, G.; Engels, M.; Benini, L.; Gyselinckx, B. (2008). A High-Performance Wireless Sensor Node for Industrial Control Applications, Third International Conference on Systems, ICONS 2008, pp. 235 - 240, 13-18 April 2008

Paulraj, A. J.; Gore, D. A.; Nabar, R. U.; Boelcskei, H. (2004). An Overview of MIMO Communications - A Key to Gigabit Wireless. Proceedings of the IEEE 92(2), 198-218.

Pellegrini, F. D. ; Miorandi, D. ; Vitturi, S.; Zanella, A. (2006). On the Use of Wireless Networks at Low Level of Factory Automation Systems, IEEE Trans. on Industrial Informatics, vol. 2, no. 2, pp. 129-143, May 2006.

Rappaport, T. S. (2002): Wireless Communications - Principles and Practice. Upper Saddle River, NJ 07458 : Prentice Hall PTR, 2002

Rappaport, T. S.; Mcgillem, C. D. (1989): UHF Fading in Factories. In: IEEE Journal on Selected Areas in Communication 7 (1989), Jan., Nr. 1, S. 40-48

Rappaport, T. S. (1989a): Indoor Radio Communications for Factories of the Future. In: IEEE Communication Magazine 27 (1989), Mai, S. 15-24

Rappaport, T. S. (1989b): Characterization of UHF Multipath Radio Channels in Factory Buildings. In: IEEE Transactions on Antennas and Propagation 37 (1989), Aug., Nr. 8, S.1058-1069

Scheible, G.; Dacfey Dzung; Endresen, J.; Frey, J.-E. (2007). Unplugged but connected - Design and Implementation of a Truly Wireless Real-Time Sensor/Actuator Interface, Industrial Electronics Magazine, IEEE, Volume: 1, Is-sue: 2, pp 25-34, 2007

Todd, S.; El-Tanny, M.; Mahmoud, S. (1992). Space and Frequency Diversity Measurements of the 1.7GHz Indoor Radio Channel Using a Four-Branch Receiver, IEEE Transactions on Vehicular Technology, Vol. 41, No 3. August 1992

Tsertou, A.; Laurenson, D. (2008). Revisiting the Hidden Terminal Problem in a CSMA/CA Wireless Network, Mobile Computing, IEEE Transactions on, Volume: 7, Issue: 7, pp. 817831,2008

VDI (2008) FA 5.21, Draft VDI/VDE-Directive 2185 : Wireless Communication in the Automation Technology - coexistence management of wireless solutions (in german), Beuth Verlag, Berlin. 
Vedral, A.; Wollert, J. F.; Buda, A.; Altrock, R. (2006). The Capability of Bluetooth for RealTime Transmission in Automation, in Proceedings of the IASTED Network and Communication Systems (NCS 2006), March 2006, pp. 168-175.

Vedral, A.; Wollert, J. F. (2006). Analysis of Error and Time Behavior of the IEEE 802.15.4 PHY-Layer in an Industrial Environment, in Proceedings of the IEEE Workshop on Factory Communication Systems (WFCS 2006), Jun. 2006, pp. 119-124.

Vedral, A. (2007). Digital Analysis, Performance Evaluation and Generative Modelling of WPAN Connections under Industrial Propagation Conditions (in german), PhD thesis, Technical University of Brandenburg in Cottbus, 2007.

Vedral, A.; Kruse, T.; Wollert, J. F. (2007). Development and performance evaluation of an antenna diversity module for industrial communication based on IEEE 802.15.4, in the proceedings of 12th IEEE International Conference on Emerging Technologies \& Factory Automation (ETFA 2007), pp. 177 - 186, Sept. 2007.

Willig, A.; Matheus, K.; Wolisz, A. (2005). Wireless Technology in Industrial Networks, Proceedings of the IEEE, vol. 93, no. 6, pp. 1130-1151, 2005.

Willig, A. (2008). How to exploit spatial diversity in wireless industrial networks, Fieldbuses and Networks in Industrial and Embedded Systems, Volume \#7, Part\#1, 2008.

Winters, J.H. (1984). Optimum Combining in Digital Mobile Radio with Cochannel Interference. IEEE Journal on Selected Areas in Communications, 2(4):528-539, July 1984.

Zepernick, H.-J., Wysocki, T.A. (1999). Multipath channel parameters for the indoor radio at 2.4 GHz ISM band, Vehicular Technology Conference, 1999 IEEE 49th, vol.1, no.pp.190-193 vol.1, July 1999.

ZigBee Standards Organization (2007), ZigBee Specification, 2007.

Zhang, K.; Zhang, D.; Jiang, W. (2008). Mitigation of Exposed Terminal Problem Using Packet Sensing, CNSR, pp 263-269, 2008 


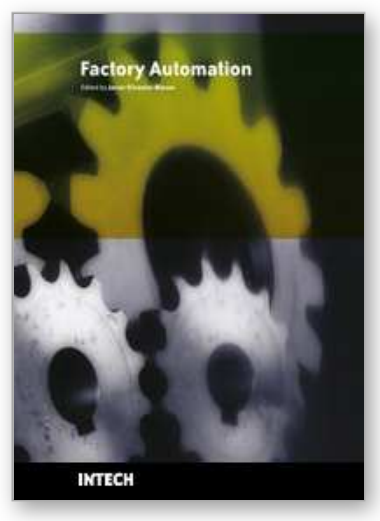

\author{
Factory Automation \\ Edited by Javier Silvestre-Blanes
}

ISBN 978-953-307-024-7

Hard cover, 602 pages

Publisher InTech

Published online 01, March, 2010

Published in print edition March, 2010

Factory automation has evolved significantly in the last few decades, and is today a complex, interdisciplinary, scientific area. In this book a selection of papers on topics related to factory automation is presented, covering a broad spectrum, so that the reader may become familiar with the various fields, and also study them in more depth where required. Within various chapters in this book, special attention is given to distributed applications and their use of networks, since it is one of the most relevant subjects in the evolution of factory automation. Different Medium Access Control and networks are analyzed, while Ethernet and Wireless networks are looked at in more detail, since they are among the hottest topics in recent research. Another important subject is everything concerning the increase in the complexity of factory automation, and the need for flexibility and interoperability. Finally the use of multi-agent systems, advanced control, formal methods, or the application in this field of RFID, are additional examples of the ideas and disciplines that experts around the world have analyzed in their work.

\title{
How to reference
}

In order to correctly reference this scholarly work, feel free to copy and paste the following:

Aurel Buda, Volker Schuermann and Joerg F. Wollert (2010). Wireless Technologies in Factory Automation, Factory Automation, Javier Silvestre-Blanes (Ed.), ISBN: 978-953-307-024-7, InTech, Available from: http://www.intechopen.com/books/factory-automation/wireless-technologies-in-factory-automation

\section{INTECH}

open science | open minds

\section{InTech Europe}

University Campus STeP Ri

Slavka Krautzeka 83/A

51000 Rijeka, Croatia

Phone: +385 (51) 770447

Fax: +385 (51) 686166

www.intechopen.com

\section{InTech China}

Unit 405, Office Block, Hotel Equatorial Shanghai No.65, Yan An Road (West), Shanghai, 200040, China 中国上海市延安西路65号上海国际贵都大饭店办公楼405单元 Phone: +86-21-62489820

Fax: $+86-21-62489821$ 
(C) 2010 The Author(s). Licensee IntechOpen. This chapter is distributed under the terms of the Creative Commons Attribution-NonCommercialShareAlike-3.0 License, which permits use, distribution and reproduction for non-commercial purposes, provided the original is properly cited and derivative works building on this content are distributed under the same license. 